\title{
HIGH-ENERGY DIFFRACTION AND DUALITY RELATIONS
}

\author{
László Jenkovszky ${ }^{1}$, István Szanyi ${ }^{2}$ \\ ${ }^{1}$ Bogolyubov Institute for Theoretical Physics, Nat. Ac. Sc. of Ukraine, Kiev, \\ jenk@bitp.kiev.ua \\ ${ }^{2}$ Uzhgorod National University, Uzhgorod, sz.istvan03@gmail.com
}

ABSTRACT. Relations between elastic and inelastic diffraction due to Regge factorisation and pomeron dominance are discussed in the light of the new phenomena discovered at the LHC in nearly forward scattering.

\section{Introduction}

At the LHC, in the nearly forward direction for the first time one can see the isolated pomeron, uncontaminated by secondary (non-leading) reggeon contributions. Elastic proton-proton scattering, single and double proton diffraction dissociation are related by factorization, as shown in Fig. 1. The input pomeron is a double pole in the $j$ plane, lying on a non-linear trajectory. Deviation from the exponential diffraction cone are studied in details.

\section{Elastic scattering}

The scattering amplitude is a sum of four terms, two asymptotic (pomeron $(P)$ and odderon $(O)$ ) and two non-asymptotic ones (secondary Regge pole contributions). $P$ and $f$ have positive $C$-parity, thus entering in the scattering amplitude with the same sign in $p p$ and $\bar{p} p$ scattering, while the odderon and $\omega$ have negative $C$-parity, thus entering $p p$ and $\bar{p} p$ scattering with opposite signs, as shown below:

$A(s, t)_{p p}^{\bar{p} p}=A_{P}(s, t)+A_{f}(s, t) \pm\left[A_{\omega}(s, t)+A_{O}(s, t)\right]$,

where the symbols $P, f, O, \omega$ stand for the relevant Regge-pole amplitudes and the super(sub)script, evidently, indicate $\bar{p} p(p p)$ scattering with the relevant choice of the signs in the sum (1). This sum can be extended by adding more reggeons, whose role may become increasingly important towards lower energies; their contribution can be effectively absorbed by $f$ and $\omega$.

We treat the odderon, $C$-odd counterpart of the pomeron on equal footing, differing by its $C$ - parity and the values of its parameters (to be fitted to the data).

The main subject of our study is the pomeron, and it is a double pole, or DP $[1,2]$ lying on a non-linear trajectory, whose intercept is slightly above one. This choice is motivated by unique properties of the DP: it produces logarithmically rising total cross sections at unit pomeron intercept. The DP satisfies unitarity by reproducing itself (scaling) under $s$-channel unitarization. By letting $\alpha_{P}(0)>1$, we allow for a faster rise of the total cross section ${ }^{1}$, although the intercept is about half that of the DL model [3] since the double pole (or dipole) itself drives the rise in energy. Due to its geometric form (see below) the DP reproduces itself against unitarity (eikonal) corrections. As a consequence, these corrections are small, and one can use the model at the "Born level" without complicated (and ambiguous) unitarity (rescattering) corrections. DP combines the properties of Regge poles and of the geometric approach.

Regge trajectories are non-linear complex functions. In a limited range and with limited precision, they can be approximated by linear trajectories (which is a common practice, reasonable when non-linear effects can be neglected). This non-linearity is manifest e.g. as the "break" i.e. a change of the slope $\Delta B \approx 2 \mathrm{GeV}^{-2}$ around $t \approx-0.1 \mathrm{GeV}^{2}$ and at large $|t|$, beyond the second maximum, $|t|>2 \mathrm{GeV}^{2}$, where the cross section flattens and the trajectories are expected to slow down logarithmically.

A simple mechanism of the diffractive dip-bump structure combining geometrical features and Regge behaviour was suggested in [2]. In that model, the dip is generated by the pomeron contribution. The relevant pomeron is a double pole arises from the interference between this dipole with a simple one, it is accompanied by. The dip-bump in the model shows correct dynamics, that is it develops from a shoulder, progressively deepening in the ISR energy region. As energy increases further, the dip is filled by the odderon contribution. At low energies the contribution from

\footnotetext{
${ }^{1} \mathrm{~A}$ supercritical pomeron trajectory, $\alpha_{P}(0)>1$ in the DP is required by the observed rise of the ratio $\sigma_{e l} / \sigma_{t o t}$, or, equivalently, departure from geometrical scaling.
} 

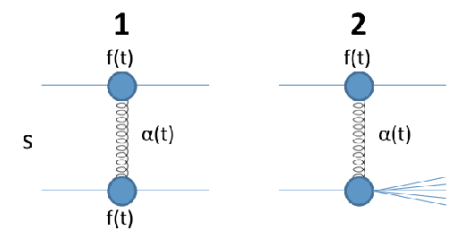

3
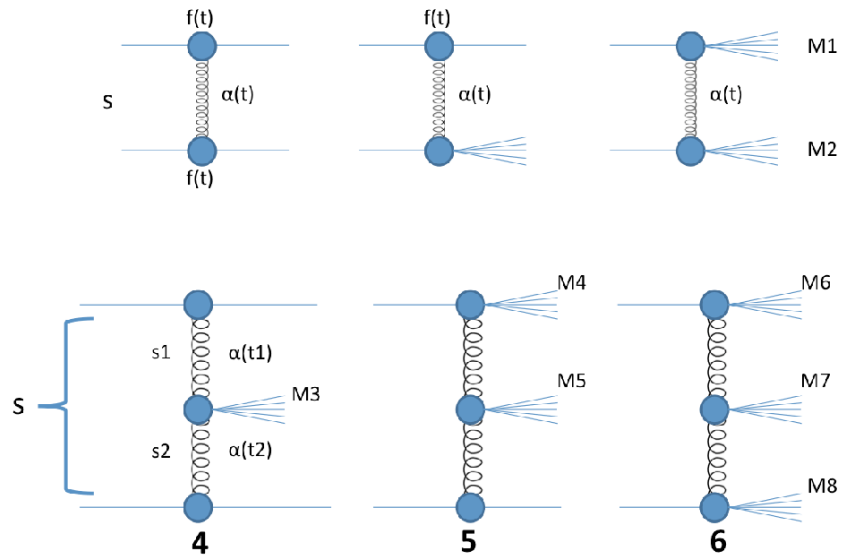

Figure 1: Diagrams for elastic scattering and diffraction dissociation (single, double and central).

non-leading, "secondary" reggeons is also present.

The dipole pomeron produces logarithmically rising total cross sections and nearly constant ratio of $\sigma_{e l} / \sigma_{t o t}$ at unit pomeron intercept, $\alpha_{P}(0)=1$. While a mild, logarithmic increase of $\sigma_{t o t}$ does not contradict the data, the rise of the ratio $\sigma_{e l} / \sigma_{t o t}$ beyond the SPS energies requires a supercritical DP intercept, $\alpha_{P}(0)=1+\delta$, where $\delta$ is a small parameter $\alpha_{P}(0) \approx 0.05$. Thus DP is about "twice softer" then that of Donnachie-Landshoff's model [3], in which $\alpha_{P}(0) \approx 0.08$.

We use the normalization where

$$
\frac{d \sigma}{d t}=\frac{\pi}{s^{2}}|A(s, t)|^{2} \text { and } \sigma_{t o t}=\left.\frac{4 \pi}{s} \Im m A(s, t)\right|_{t=0} .
$$

Neglecting spin dependence, the invariant proton(antiproton)-proton elastic scattering amplitude is that of Eq. (1). The secondary reggeons are parametrized in a standard way, with linear Regge trajectories and exponential residua, where $R$ denotes $f$ or $\omega$ - the principal non-leading contributions to $p p$ or $\bar{p} p$ scattering:

$$
A_{R}(s, t)=a_{R} \mathrm{e}^{-i \pi \alpha_{R}(t) / 2} \mathrm{e}^{b_{R} t}\left(s / s_{0}\right)^{\alpha_{R}(t)},
$$

with $\alpha_{f}(t)=0.70+0.84 t$ and $\alpha_{\omega}(t)=0.43+0.93 t$.

The pomeron is a dipole in the $j$-plane

$$
\begin{gathered}
A_{P}(s, t)=\frac{d}{d \alpha_{P}}\left[\mathrm{e}^{-i \pi \alpha_{P} / 2} G\left(\alpha_{P}\right)\left(s / s_{0}\right)^{\alpha_{P}}\right]= \\
\mathrm{e}^{-i \pi \alpha_{P}(t) / 2}\left(s / s_{0}\right)^{\alpha_{P}(t)}\left[G^{\prime}\left(\alpha_{P}\right)+(L-i \pi / 2) G\left(\alpha_{P}\right)\right] .
\end{gathered}
$$

Since the first term in squared brackets determines the shape of the cone, one fixes

$$
G^{\prime}\left(\alpha_{P}\right)=-a_{P} \mathrm{e}^{b_{P}\left[\alpha_{P}-1\right]},
$$

where $G\left(\alpha_{P}\right)$ is recovered by integration, and, as a consequence, the pomeron amplitude Eq. (4) can be rewritten in the following "geometrical" form

$$
A_{P}(s, t)=i \frac{a_{P} s}{b_{P} s_{0}}\left[r_{1}^{2}(s) \mathrm{e}^{r_{1}^{2}(s)\left[\alpha_{P}-1\right]}-\varepsilon_{P} r_{2}^{2}(s) \mathrm{e}^{r_{2}^{2}(s)\left[\alpha_{P}-1\right]}\right]
$$

where $r_{1}^{2}(s)=b_{P}+L-i \pi / 2, \quad r_{2}^{2}(s)=L-i \pi / 2, \quad L \equiv$ $\ln \left(s / s_{0}\right)$.

The main features of the nonlinear trajectories are: 1) presence of a threshold singularity required by $t$-channel unitarity and responsible for the change of the slope in the exponential cone (the so-called "break") near $t=-0.1 \mathrm{GeV}^{2}$ [4], and 2) logarithmic asymptotic behaviour providing for a power fall-off of the cross sections in the "hard" region. The combination of theses properties, however is not unique.

\subsection{Diffraction minimum (dip-bump)}

Fig. 2 (a) shows the $p p$ and $\bar{p} p$ total elastic scattering cross section calculated from the model (values of parameters see in [5]). Figure 2 (b) shows the ratio of the real to imaginary part of the forward amplitude. The model with a linear trajectory describes reasonably the data a wide range of collision energies for $p p$ and $\bar{p} p$. Figures 3 (a,b,c) show the fitted $\bar{p} p$ and $p p$ differential cross sections and predictions for three different center of mass energies are shown. The yellow area exhibits the statistical uncertainty on the calculations, described earlier. Calculations are characterized by an approximately exponential fall-off in the range $0<|t|<8 \mathrm{GeV}^{2}$, with the slope changing around $-t \approx 0.6 \mathrm{GeV}^{2}$. The dip moved towards lower momentum transfer and became almost filled by the odderon. 


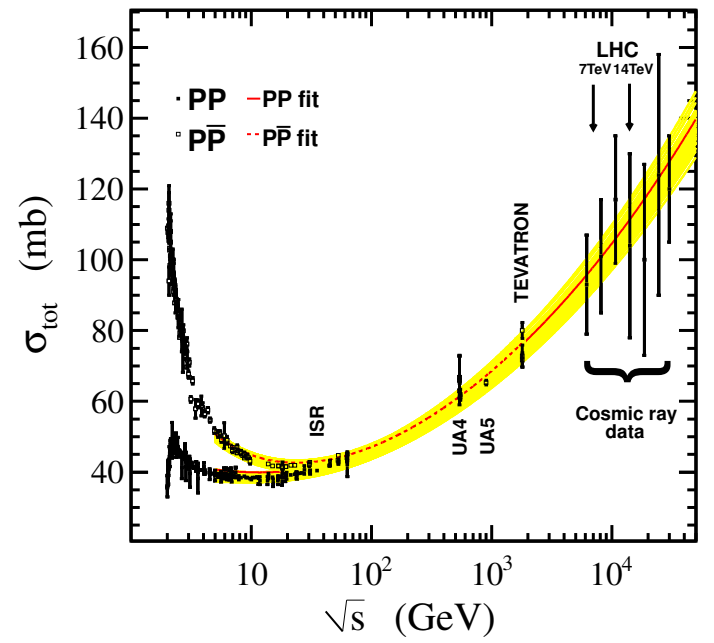

(a)

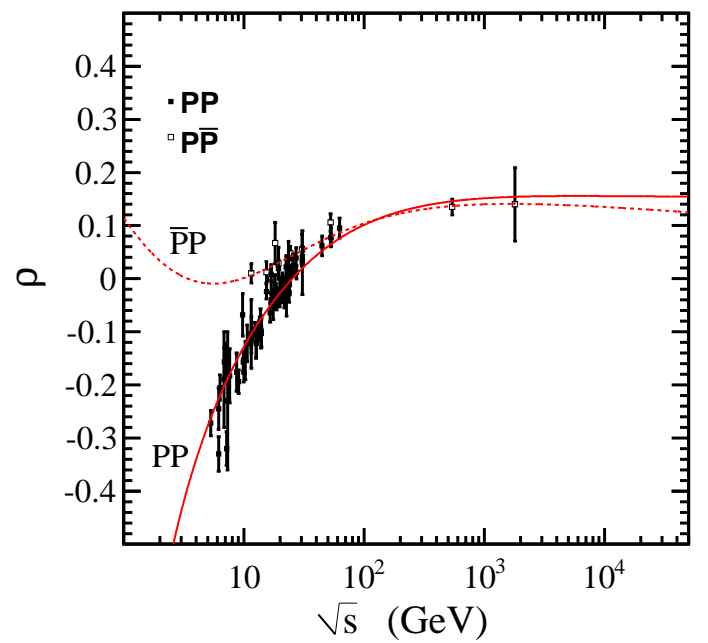

(b)

Figure 2: (a) Total $p p$ and $\bar{p} p$ cross sections calculated from the model, fitted to the data in the range $\sqrt{s}=5$ - $30 \mathrm{TeV}$ and $5 \mathrm{GeV}-1.8 \mathrm{TeV}$, respectively. (b) Ratio of the real to imaginary part of the forward amplitude for $p p$ and $\bar{p} p$, calculated from the model and fitted to the data.

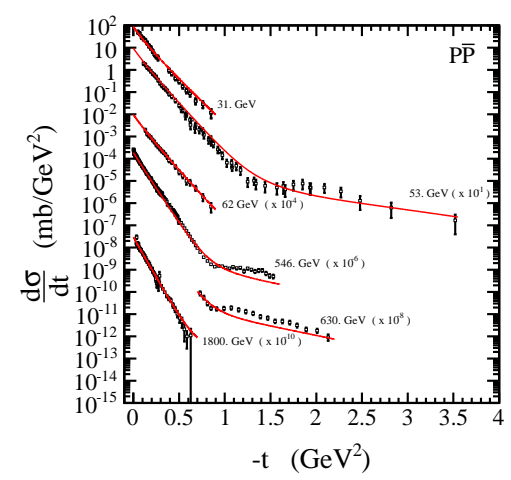

(a)

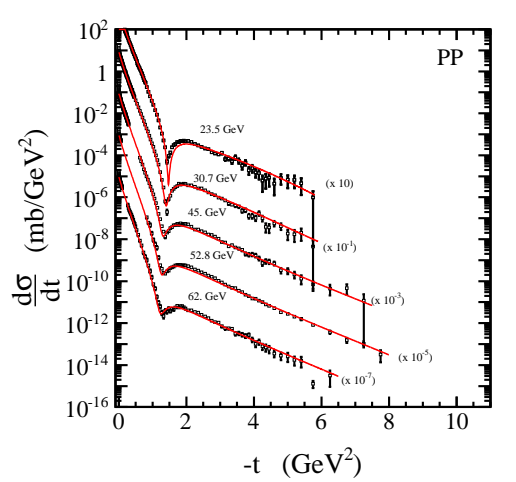

(b)

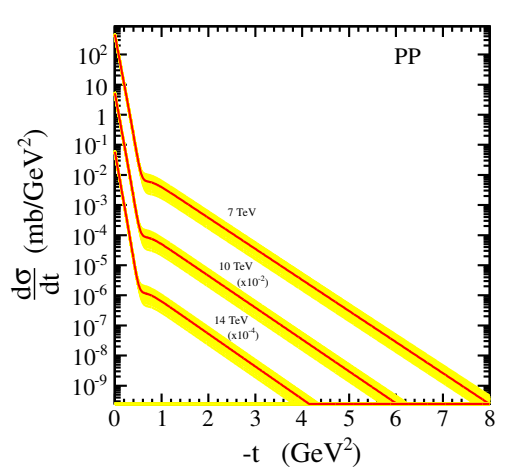

(c)

Figure 3: (a) $\bar{p} p$ differential cross sections calculated from the model, fitted to the data in the range $-t=0.1$ $-8 \mathrm{GeV}^{2}$. (b) $p p$ differential cross sections calculated from model fitted to the data. (c) predictions for the $p p$ differential cross section calculated from the model for three different LHC energies. 


\subsection{Low- $|t|$ "break" and proton shape}

The diffraction cone of high-energy elastic hadron scattering deviates from a purely exponential dependence on $t$ due to two structures clearly visible in proton-proton scattering: the so-called "break" (in fact, a smooth curve with a concave curvature) near $t=-0.1 \mathrm{GeV}^{2}$, whose position is nearly independent of energy and the prominent "dip" - diffraction minimum, moving slowly (logarithmically) with $s$ towards smaller values of $|t|$, where $s$ and $t$ are the Mandelstam variables. The physics of these two phenomena are quite different. As illustrated in Fig. 4, the "break" is likely a reflection due to the "pion cloud", which controls the "static size" of nucleon. This effect, first observed in 1972 at the ISR, was interpreted $[4,6,7]$ as the manifestation of $t$-channel unitarity, generating a two-pion loop in the cross channel, Fig. 5, and was referred to by Bronzan [8] as the "fine structure" of the pomeron. The dip (diffraction minimum), on the other hand, is generally accepted as a consequence of $s$ channel unitarity or absorption corrections to the scattering amplitude. As such, dip location reflects the "size" of proton, moving towards smaller $|t|$ values as the total cross section increases with energy.

The departure from the linear exponential behavior was confirmed by recent measurements by the TOTEM Collaboration at the CERN LHC, first at $8 \mathrm{TeV}$ (with a significance greater then $7 \sigma$ ) [9] and subsequently at $13 \mathrm{TeV}$ [10]. At the ISR the "break" was illustrated by plotting the local slope $B(t)$ for several $t$-bins at fixed values of $s$.

At the LHC the effect appears of the same order of magnitude and is located near the same value of $t$. Different from the ISR [11], TOTEM quantifies the deviation from the exponential by normalizing the measured cross section to a linear exponential form, (see Eq. (9) below). For the sake of completeness we will exhibit this "break effect" both in the normalized form and for $B(t)$.

The new LHC data from TOTEM at $8 \mathrm{TeV}$ confirm the conclusions $[4,6,7]$ about the nature of the break and call for a more detailed analysis and better understanding of this phenomenon. The new data triggered intense theoretical work in this direction [14, 13, 12], but many issues still remain open. Although the curvature for $B(t)$, both at the ISR and the LHC is concave, a convex behavior cannot be excluded in other reactions and/or new energies. While the departure from a linear exponential was studied in details both at the ISR and LHC energies, an interpolation between the two is desirable to clarify the uniqueness of the phenomenon. This is a challenge for the theory, and it can be done within Regge-pole models. Below we do so by adopting a simple Regge-pole model, with a pomeron and two secondary reggeons, $f$ and $\omega$ exchanges. The odderon may also be present. However its contribution at low $|t|$ is too small to be identified unambiguously.

Having identified [4, 6, 7] the observed "break" with a two-pion exchange effect, we investigate further two aspects of the phenomenon, namely: 1) to what extent is the "break" observed recently at the LHC a "recurrence" of that seen at the ISR (universality)? 2) what is the relative weight of the Regge residue (vertex) compared to the trajectory (propagator) in producing the "break"? We answer these questions by means of a detailed fit to the elastic proton-proton scattering data in the relevant kinematic range $0.05<-t<0.35 \mathrm{GeV}^{2}$ ranging between the ISR energies $(23.5 \leq \sqrt{s} \leq 62.5$ $\mathrm{GeV}$ ), and those available at the LHC.

As shown by Barut and Zwanziger [15], $t$-channel unitarity constrains the Regge trajectories near the threshold, $t \rightarrow t_{0}$ by

$$
\Im \alpha(t) \sim\left(t-t_{0}\right)^{\Re \alpha\left(t_{0}\right)+1 / 2},
$$

where $t_{0}$ is the lightest threshold, $4 m_{\pi}^{2}$ in the case of the vacuum quantum numbers (pomeron or $f$ meson). Since the asymptotic behavior of the trajectories is constrained by dual models with Mandelstam analyticity by square-root (modulus $\ln t$ ): $\left|\frac{\alpha(t)}{\sqrt{t} \ln t}\right|_{t \rightarrow \infty} \leq$ const, (see [4] and references therein), for practical reasons it is convenient to approximate, for the region of $t$ in question, the trajectory as a sum of square roots. Higher thresholds, indispensable in the trajectory, may be approximated by their power expansion, i.e. by a linear term, matching the threshold behavior with the asymptotic.

At the ISR, the proton-proton differential cross section was measured at $\sqrt{s}=23.5,30.7,44.7,52.8$ and $62.5 \mathrm{GeV}$ [16]. In all the above energies the differential cross section changes its slope near $-t=0.1 \mathrm{GeV}^{2}$. By using a simple Regge-pole model we have mapped the "break" fitted at the ISR onto the LHC TOTEM 8 TeV data. The simple Regge-pole model is constructed by a leading supercritical pomeron and two secondary, $f$ and $\omega$ contributions.

The detailed results of fits and the parameters see in [17]. To demonstrate the important features more clearly, we show the results of the mapping in higher resolution in Fig. 6 and Fig. 7. In Fig. 6, we exhibit the shape of local slopes, defined by

$$
B(s, t)=\frac{d}{d t} \ln (d \sigma / d t)
$$

at $\operatorname{six} s$ values. To better demonstrate the quality of our fit and to anticipate the comparison with the LHC data, we also present in Fig. 7 the ISR data in normalized form as used by TOTEM [9]:

$$
R=\frac{d \sigma / d t}{d \sigma / d t_{\text {ref }}}-1,
$$

where $d \sigma / d t_{r e f}=A e^{B t}$, with $A$ and $B$ constants determined from a fit to the experimental data. 


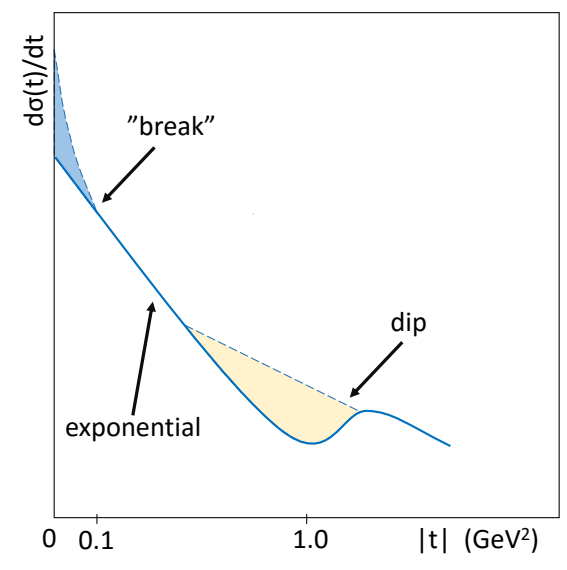

(a)

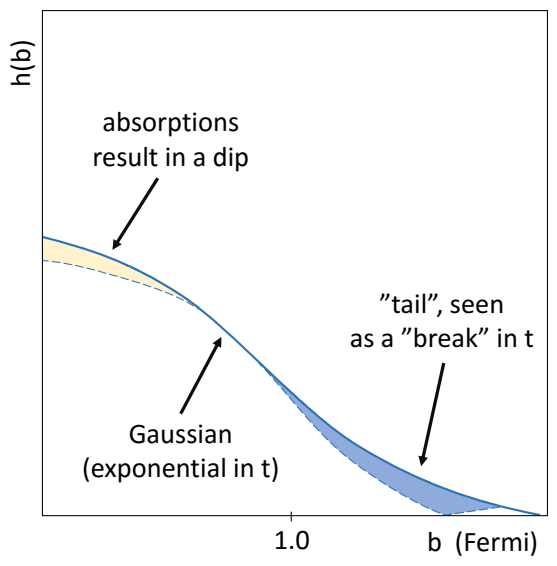

(b)

Figure 4: The "break" followed by a single diffraction minimum ("dip") and maximum, shown both as function in $t$ and its Fourier transform (impact parameter representation), in $b$.
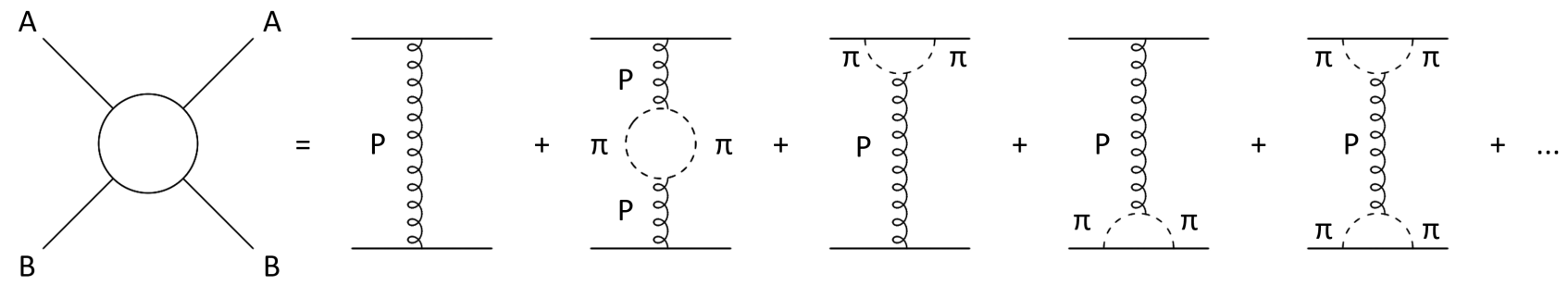

Figure 5: Diagram for elastic scattering with $t$-channel exchange containing a branch point at $t=4 m_{\pi}^{2}$.

Both Fig. 6 and Fig. 7 re-confirm the earlier finding that the "break" can be attributed the presence of two-pion branch cuts in the Regge parametrization. The effect of pion cloud manifest itself most importantly through the Regge residue, rather then the pomeron trajectory.

\section{Diffraction dissociation, single (SD) and double (DD)}

In most of the papers SD is calculated from the triple Regge limit of an inclusive reaction, as shown in Fig. 8.

In that limit, the double diffraction cross section can be written as

$$
\frac{d^{2} \sigma}{d t d M_{x}^{2}}=\frac{G_{13,2}^{P P, P(t)}}{16 \pi^{2} s_{0}^{2}}\left(\frac{s}{s_{0}}\right)^{2 \alpha_{P}(t)-2}\left(\frac{M^{2}}{s_{0}}\right)^{\alpha_{P}(0)-\alpha_{P}(t)} .
$$

This approach has two shortcomings. The first one is that it leaves outside the small- $M^{2}$ resonance region. The second one is connected with the fact that whatever the pomeron, the (partial) SD cross section overshoots the total one, thus obviously conflicting with unitarity. Various ways of resolving this deficiency are known from the literature, including the vanishing (decoupling) of the triple pomeron coupling, but none of them can be considered completely satisfactory.
We instead follow the idea [18] according to which the reggeon (here, the pomeron) is similar to the photon and that the reggeon-nucleon interaction is similar to deep-inelastic photon-nucleon scattering (DIS), with the replacement $-Q^{2}=q^{2} \rightarrow t$ and $s=W^{2} \rightarrow M_{x}^{2}$. There is an obvious difference between the two: while the $C$ parity of the photon is negative, it is positive for the pomeron. We believe that while the dynamics is essentially invariant under the change of $C$, the difference between the two being accounted for by the proper choice of the parameters. Furthermore, while Jaroszewicz and Landshoff [14], in their pomeronnucleon DIS structure function (SF) (or $P p$ total cross section) use the Regge asymptotic limit, we include also the low missing mass, resonance behaviour.

It is evident that Regge factorization is essential in both approaches (triple Regge and the present one). It is feasible when Regge singularities are isolated poles. While the pre-LHC data require the inclusion of secondary reggeons, at the $\mathrm{LHC}$ we are in the fortunate situation of a single pomeron exchange (pomeron dominance) in the $t$ channel in single and double diffraction (not necessarily so in central diffraction, to be treated elsewhere). Secondary Regge pole exchanges will appear however, in our dual-Regge treatment of $P p$ scattering (see below), not to be confused with the the $t$ channel of $p p$. This new situation makes diffraction 


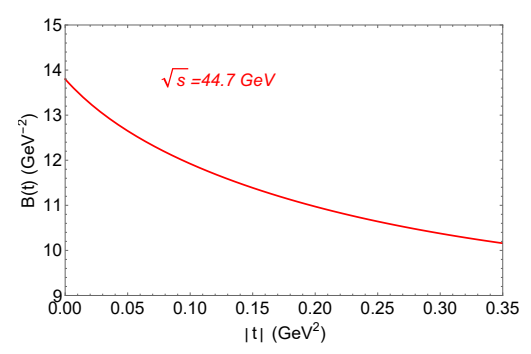

(a) $44.7 \mathrm{GeV}$

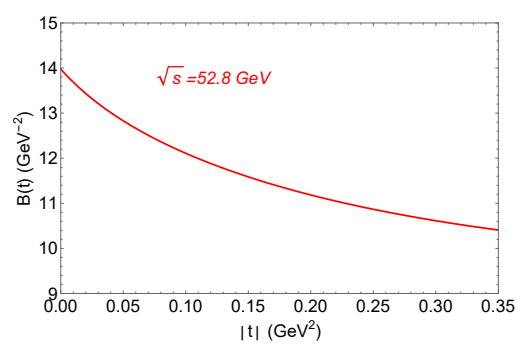

(b) $52.8 \mathrm{GeV}$

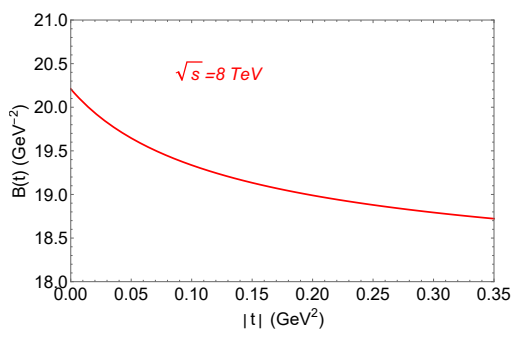

(c) $8 \mathrm{TeV}$

Figure 6: Local slopes.

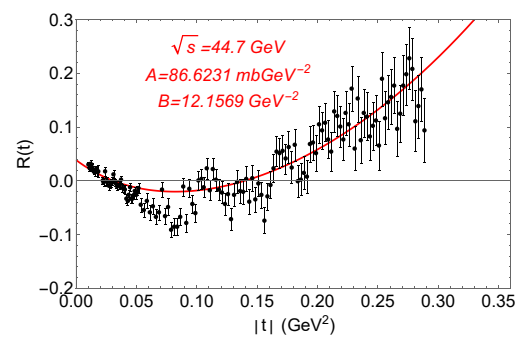

(a) $44.7 \mathrm{GeV}$

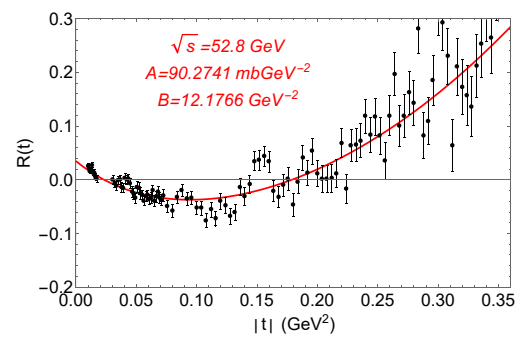

(b) $52.8 \mathrm{GeV}$

Figure 7: $R(t)$ ratios.

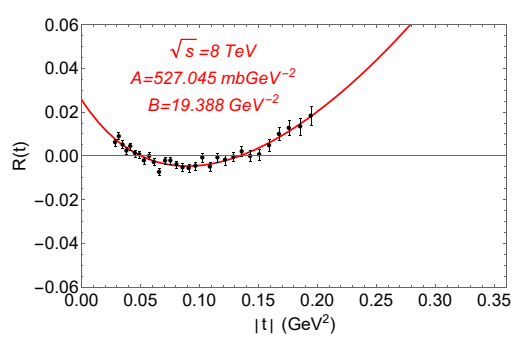

(c) $8 \mathrm{TeV}$
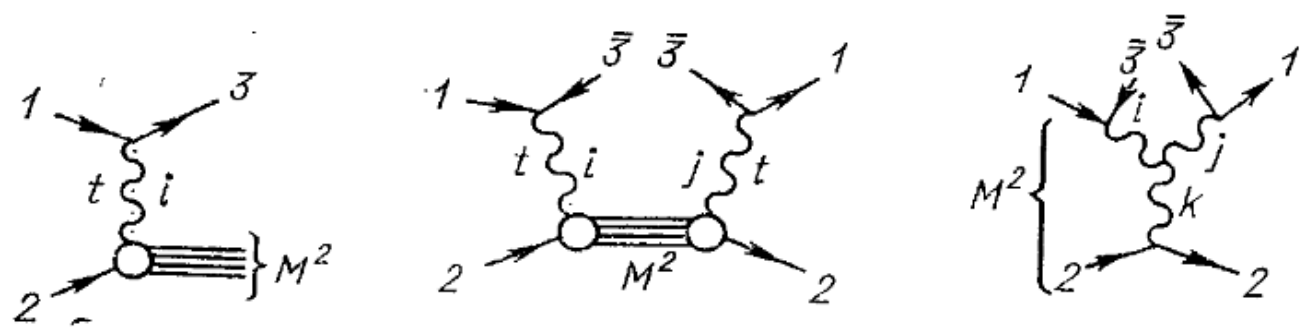

Figure 8: From SD to the triple Regge limit. 
at the LHC unique in the sense that for the first time Regge-factorization is directly applicable. We make full use of it.

Diffraction is limited both in the missing mass (coherence), $\xi \lesssim 0.05$ and in $t$ ("soft" collisions). There is a transition region in $t$ from "soft" to "hard" collisions, with a possible dip-bump structure between the two. To be sure, in our analysis we leave outside these interesting but controversial points, concentrating on the "first" cone with clear exponential behaviour.

With the advent of the LHC, diffraction, elastic and inelastic scattering entered a new area, where it can be seen uncontaminated by non-diffraction events. In terms of the Regge-pole theory this means, that the scattering amplitude is completely determined by a pomeron exchange, and in a simple-pole approximation, Regge factorization holds and it is of practical use!

\subsection{A compilation of basic formulae}

This subsection contains a compilation of the main formulae used in the calculations and fits to the data.

The single diffraction (SD) dissociation cross section is:

$$
2 \cdot \frac{d^{2} \sigma_{S D}}{d t d M_{x}^{2}}=F_{p}^{2}(t) F_{\text {inel }}^{2}\left(t, M_{x}^{2}\right)\left(\frac{s}{M_{x}^{2}}\right)^{2(\alpha(t)-1)} .
$$

Double diffraction (DD) dissociation cross section:

$$
\begin{aligned}
& \frac{d^{3} \sigma_{D D}}{d t d M_{1}^{2} d M_{2}^{2}}=N_{D D} F_{\text {inel }}^{2}(\left.t, M_{1}^{2}\right) F_{\text {inel }}^{2}\left(t, M_{2}^{2}\right) \times \\
& \times\left(\frac{s s_{0}}{M_{1}^{2} M_{2}^{2}}\right)^{2(\alpha(t)-1)} .
\end{aligned}
$$

with the norm $N_{D D}=\frac{1}{4 A_{e l}}$, and the inelastic vertex:

$$
F_{\text {inel }}^{2}\left(t, M_{x}^{2}\right)=A_{r e s} \frac{1}{M_{x}^{4}} \sigma_{T}^{P p}\left(M_{i}^{2}, t\right)+C_{b g} \sigma_{B g},
$$

where the pomeron-proton total cross section is the sum $N^{*}$ resonances and the Roper resonance, with a relevant norm factor $R$ (we remove the $t$ dependent $f_{\text {res }}(t)$ out of the sum):

$$
\begin{gathered}
\sigma_{T}^{P p}\left(M_{x}^{2}, t\right)=R \frac{\left[f_{\text {res }}(t)\right]^{2} \cdot M_{\text {Roper }}\left(\frac{\Gamma_{\text {Roper }}}{2}\right)}{\left(M_{x}^{2}-M_{\text {Roper }}^{2}\right)^{2}+\left(\frac{\Gamma_{\text {Roper }}}{2}\right)^{2}}+ \\
+\left[f_{\text {res }}(t)\right]^{4} \sum_{n=1,3} \frac{\mathcal{I} m \alpha\left(M_{x}^{2}\right)}{\left(2 n+0.5-\mathcal{R} e \alpha\left(M_{x}^{2}\right)\right)^{2}+\left(\mathcal{I} m \alpha\left(M_{x}^{2}\right)\right)^{2}},
\end{gathered}
$$

and the background corresponding to non-resonance contributions:

$$
\sigma_{B g}=\frac{f_{b g}(t)}{\frac{1}{\left(M_{x}^{2}-\left(m_{p}+m_{\pi}\right)^{2}\right)^{\varsigma}}+\left(M_{x}^{2}\right)^{\eta}},
$$

Integrated cross sections are calculated as:

$$
\frac{d \sigma_{S D}}{d t}=\int_{M_{1}^{2}}^{M_{2}^{2}} \frac{d^{2} \sigma_{S D}}{d t d M_{x}^{2}} d M_{x}^{2}
$$

for SD and:

$$
\frac{d \sigma_{D D}}{d t}=\iint_{f\left(M_{x_{1}}^{2}, M_{x_{2}}^{2}\right)} \frac{d^{3} \sigma_{S D}}{d t d M_{x_{1}}^{2} d M_{x_{2}}^{2}} d M_{x_{1}}^{2} d M_{x_{2}}^{2}
$$

for DD.

We calculate also fully integrated cross sections:

$$
\begin{gathered}
\sigma_{S D}=\int_{0}^{1} d t \int_{M_{t h}^{2}}^{0.05 s} d M_{x}^{2} \frac{d^{2} \sigma_{S D}}{d t d M_{x}^{2}} \\
\sigma_{D D}=\int_{0}^{1} d t \iint_{\Delta \eta>3} d M_{x_{1}}^{2} d M_{x_{2}}^{2} \frac{d^{3} \sigma_{D D}}{d t d M_{x_{1}}^{2} d M_{x_{2}}^{2}}
\end{gathered}
$$

and

$$
\frac{d^{2} \sigma_{D D}}{d M_{x_{1}}^{2} d M_{x_{2}}^{2}}=\int_{0}^{1} \frac{d^{3} \sigma_{D D}}{d t d M_{x_{1}}^{2} d M_{x_{2}}^{2}} d t
$$

\subsection{Fitting procedure}

The model contains 12 parameters, a large part of which is fixed either by their standard values (e.g. those of Regge trajectories, except for the pomeron slope, whose slope exceeds the "standard" value to meet the SD data) or are set close to the previous fits [18].

The elastic data and Regge theory fix the parameters $s_{0}, \alpha(0), \alpha^{\prime}, A_{e l}, b_{e l}$.

The data at larger $|t|$, with the dip-bump structure and subsequent flattening of the cross section, both in elastic scattering and in SD may indicate the onset of new physics and the transition to hard scattering, implying a non-exponential residue and/or a non-linear pomeron trajectory, that goes beyond the present study.

Single diffraction dissociation (SD) is an important pillar in our fitting procedure. The following parameters were fitted to the SD data: $A_{r e s}, C_{b g}, R, b_{r e s}$, $b_{b g}, \varsigma, \eta$. As input data we use double differential cross sections $\frac{d^{2} \sigma_{S D}}{d t d M^{2}}$ versus $M_{x}^{2}$ at $|t|=0.05 \mathrm{GeV}^{2}$; b)single differential cross sections $\frac{d \sigma_{S D}}{d t}$ vs. $t$; c) fully integrated cross sections versus energy $\sqrt{s}$.

Central exclusive diffraction (Diagram 6. in Fig. 1) was calculted recently in [19].

\section{Conclusions}

At the LHC, in the diffraction cone region $\left(t<1 \mathrm{GeV}^{2}\right)$ proton-proton scattering is dominated (over $95 \%$ ) by pomeron exchange (quantified in [5]). This enables full use of factorized Regge-pole models. 


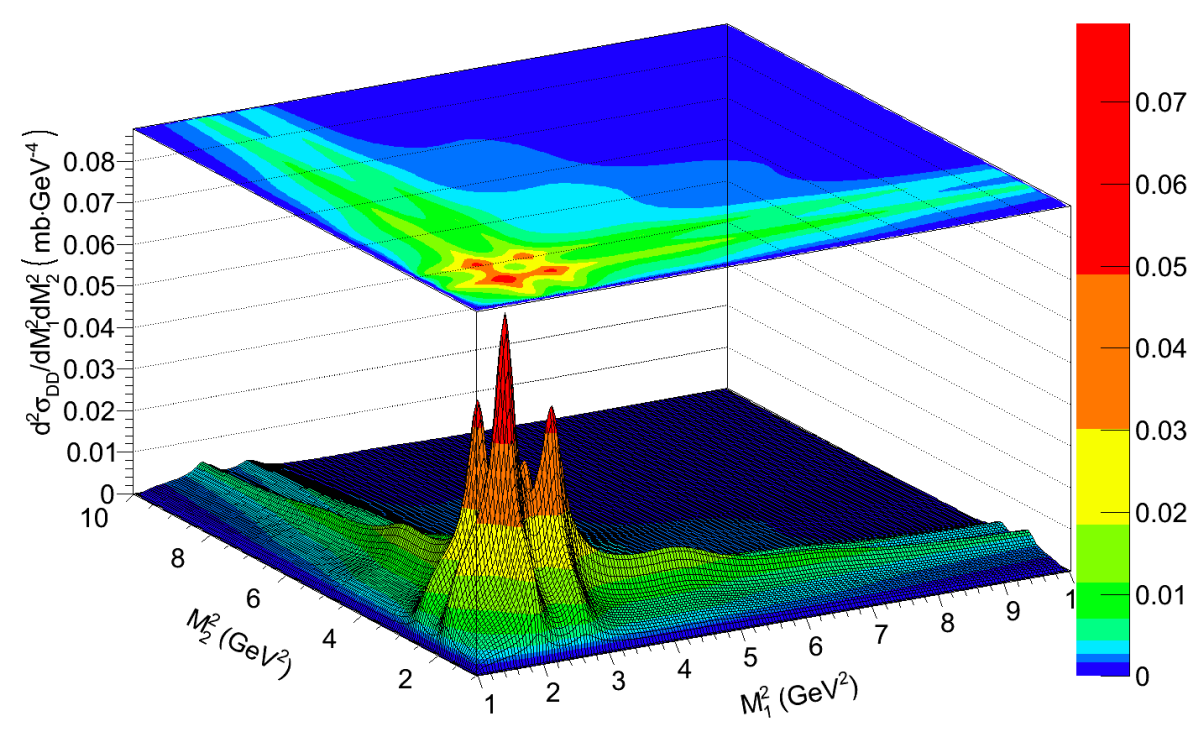

Figure 9: Double differential DD cross section as function of $M_{1}^{2}$ and $M_{2}^{2}$, integrated in $t$; , see Eq. (11).

Contributions from non-leading (secondary) trajectories can (and should be) included in the extension of the model to low energies, e.g. below those of the SPS. Acknowledgements. L.J. thanks the organizers of the Gamov Conference for their hospitality. The work of I.Sz. was supported by HDFU (UMDSZ).

\section{References}

[1] R. Fiore et al. Int. J. Mod. Phys., A24, 2551-2559 (2009); arXiv:hep-ph/0812.0539.

[2] L.L. Jenkovszky and A.N. Vall, ITP Preprint, Kiev 1974; Czech. J. Phys. B, 26, 447 (1976).

[3] S. Donnachie and P. Landshoff, Phys. Lett., B 123, 345 (1983); Nucl. Phys. 267, 690 (1985).

[4] G. Cohen-Tannoudji et al., Nuov. Cim., 5, 957 (1972).

[5] L.L. Jenkovszky, A.I. Lengyel, D.I. Lontkovsky, Int. J. Mod. Phys. A, 26, 4755 (2011); arXiv:1105.1202.

[6] C.-I Tan and D.M. Tow, Phys. Letters B. 53B, 452 (1975).

[7] U. Sukhatme, Chung-I Tan, and Tran Thanh Van, Z. Phys. C, Particles and Fields, 1, 95 (1979).

[8] J.B. Bronzan, Fine structure of the Pomeron, in Symposium on the Pomeron, ANL report, ANL/HEP 7327, p.34, (Argonne National Laboratory, 1973).
[9] TOTEM Collab. (G. Antchev et al.), Nucl. Phys., B 899527527 (2015); arXiv:1503.08111.

[10] M. Deile, Elastic and Total Cross-Section Measurements by TOTEM, Contribution at the 17. Conference on Elastic and Diffractive Scattering, (Prague, Czech Republic, 2017).

[11] B. Barbiellini et. al., Phys. Lett. B39, 663 (1972).

[12] L. Jenkovszky, I. Szanyi, Phys. Part. Nuclei Lett., 14, 687 (2017); arXiv:1701.01269.

[13] D.A. Fagundes, L. Jenkovszky, E.Q. Miranda, G. Pancheri, P.V.R.G. Silva, Int. J. Mod. Phys. A 31, 1645022 (2016); arXiv:1509.02197.

[14] L. Jenkovszky and A. Lengyel, Acta Phys. Pol., B 46, 863 (2015); arXiv:1410.4106.

[15] A.O. Barut and D.E. Zwanziger, Phys. Rev. 127, 974 (1962).

[16] http://durpdg.dur.ac.uk/review/pp2/exphtml/ CERNISR.shtml

[17] L. Jenkovszky, I. Szanyi and C.-I Tan. Shape of Proton and the Pion Cloud, arXiv:1710.10527.

[18] R. Fiore et al. Phys. Rev. D 83, 056014 (2011); arXiv:056014; R. Fiore et al., Yad. Fizika, 771535 (2014); arXiv:1011.0664.

[19] R. Fiore, L. Jenkovszky and R. Schicker, EPJ C 76(1) 1-10 (2016); arXiv:1512.04977. 